HWS/92-07, CSULB-HEP-45

\title{
Solitons and Instantons with(out) Supersymmetry
}

\author{
Zvonimir Hlousek* \\ Department of Physics and Astronomy \\ California State University, Long Beach \\ Long Beach, CA 90840 USA \\ Donald Spector ${ }^{\dagger}$ \\ Department of Physics, Eaton Hall \\ Hobart and William Smith Colleges \\ Geneva, NY 14456 USA
}

\begin{abstract}
We give model-independent arguments, valid in nearly any number of spacetime dimensions, that topological solitons and instantons satisfy Bogomol'nyi-type bounds and, when these bounds are saturated, satisfy self-duality equations. In the supersymmetric case, we also show that, in spacetime dimensions greater than two, theories with topological charges necessarily exhibit extended supersymmetry, in which the topological charge appears as the central charge. The significance of our arguments lies in their generality. In the supersymmetric case, we obtain insight into the contrast observed between topological charges in $1+1$ and higher dimensional models. The centerpiece of our method is to require that the supersymmetric extension of a generic (non-supersymmetric) field theory be selfconsistent.
\end{abstract}

11/92; revised 4/94

\footnotetext{
* hlousek@beach.csulb.edu

† spector@hws.bitnet
} 


\section{Introduction and Summary}

The topological classification of field configurations is an important ingredient in our non-perturbative understanding of field theories. Topological solitons and instantons have, in particular, received a great deal of attention. The basic theory of these field configurations is covered well in [1], which also contains a comprehensive list of references to the original literature.

The study of solitons and instantons in various models has revealed important similarities between these two types of field configurations. Solitons generally exhibit Bogomol'nyi bounds (their energy is bounded from below by the magnitude of the topological charge); instantons generally exhibit analogous bounds, with their (Euclidean) action bounded from below by the magnitude of the instanton number. When field configurations saturate such a bound, they not only automatically satisfy the equations of motion, but they also, in fact, generally satisfy simpler first-order equations, called Bogomol'nyi or self-duality equations. Perhaps the most familiar examples are the Bogomol'nyi equations [2] for the 't Hooft-Polyakov monopole [3] in the PrasadSommerfield limit 44], and the self-duality equations [5] for Yang-Mills instantons [6] in $3+1$ dimensions. We will use the phrase "Bogomol'nyi relationships" to refer collectively to Bogomol'nyi bounds and Bogomol'nyi equations.

In recent work, we began to develop a model-independent approach to demonstrating the appearance of Bogomol'nyi relationships [7] [8] [9]. That work suffered from two limitations: it was valid only in $2+1$ and $3+1$ dimensions, and it pertained only to solitons, not to instantons. In this paper, we address those shortcomings, extending our arguments to solitons in any number of spacetime dimensions, $2+1$ or higher; and to instantons in any number of Euclidean dimensions, two or higher. Our method here, as in our previous papers, is to require that the supersymmetric extension of a theory behave self-consistently. Thus, here, as an essential step, we extend our earlier work in the supersymmetric case to higher dimensions as well, showing that in $2+1$ or more dimensions, any theory with $N=1$ supersymmetry and a conserved topological charge automatically has extended supersymmetry in which the topological charge appears as the central charge.

Throughout this paper, since we are only examining topologically non-trivial soliton and instanton field configurations, we will, for simplicity, use the term "soliton" to mean any field configuration with a non-zero value of a conserved topological charge (given by an 
integral over all space), and the term "instanton" to mean any Euclidean field configuration with a non-zero value of a topological index (given by an integral over all spacetime).

Note that the importance of our work is that it provides a general explanation for properties of solitons and instantons which appear routinely but which have been understood only in a model-dependent way. Consequently, we focus here on presenting those general arguments. For specific examples, we will refer the reader to the literature, which already contains analyses of a number of models. Furthermore, since this work generalizes our earlier results, we will concentrate here on what is new, and simply summarize our earlier results as needed. The reader interested in the details of our earlier work should consult our papers listed in the references.

In the interests of clarity, we will divide the argument into two pieces, one supersymmetric and one not. In the next section, we will show that in any theory in $2+1$ or more spacetime dimensions with simple supersymmetry and a topological charge, an extended superalgebra appears, in which additionally the topological charge appears as the central charge of the extended superalgebra. Then, in the subsequent section, we will use this result as the basis for arguments that solitons and instantons in general (nonsupersymmetric) theories exhibit Bogomol'nyi bounds.

Finally, the reader is reminded that Bogomol'nyi relationships are classical statements. The extended superalgebra we uncover in the next section does appear in both the classical and quantum theories. The other results in the paper, however, which depend, for example, on the equations of motion, are to be understood as classical results only.

\section{The Argument, Part 1: Solitons and Extended Supersymmetry}

In [10], Olive and Witten discovered that in a number of models with supersymmetry and a topologically conserved charge, the full invariance algebra is actually an extended supersymmetry algebra in which the topological charge appears as the central charge. This type of structure was found again in Chern-Simons models with vortices [11], and in the $2+1$ dimensional $O(3)$ non-linear sigma model [12], and in 10-dimensional string and string-related models [13]. In [8], a general explanation for this phenomenon was obtained for $2+1$ and $3+1$ dimensional models. Here we extend that work to an arbitrary number of spacetime dimensions (greater than two), and we see explicitly why the case of $1+1$ spacetime dimensions is different. 
Consider a theory with $N=1$ supersymmetry and a conserved topological charge. When a charge is conserved topologically, this means that the conservation law holds without use of the equations of motion. Consequently the conserved current $J_{\mu}$ can be written as the curl of a potential [14]. In $d>2$ spacetime dimensions, this means

$$
J_{\mu_{1}}=\epsilon_{\mu_{1} \cdots \mu_{d}} \partial^{\mu_{2}} A^{\mu_{3} \cdots \mu_{d}}
$$

The potential $A$ is a $(d-2)$-index antisymmetric tensor. (We find it convenient to use tensors rather than forms.) This potential is not a fundamental field, but is an expression defined in terms of the fields of the theory. Now the potential is not uniquely defined; rather, it is only defined up to gauge transformations. Under

$$
A^{\mu_{1} \cdots \mu_{d-2}} \rightarrow A^{\mu_{1} \cdots \mu_{d-2}}+\partial^{\left[\mu_{1}\right.} \Omega^{\left.\mu_{2} \cdots \mu_{d-2}\right]}
$$

the current $J_{\mu}$ is unchanged. As we show in the appendix, there is a choice of gauge in which $A$ is divergenceless. Hereon, we use that gauge exclusively.

Now recall that we are considering a theory with $N=1$ supersymmetry. We denote the supercharges $Q_{\alpha}$, where $\alpha$ is a spinor index. Define

$$
\tilde{S}_{\mu_{1}}^{\alpha}=\left[Q^{\beta}, A_{\mu_{1} \cdots \mu_{d-2}}\right]\left(\gamma^{\mu_{2}} \cdots \gamma^{\mu_{d-2}}\right)_{\beta}^{\alpha}
$$

(Note that this expression is only sensible for $d \geq 3$.) Since the supersymmetry charges commute with the translation generators, and $A$ is divergenceless, we have that $\tilde{S}$ is a conserved spinor current. Furthermore, the supersymmetry transform of $\tilde{S}$ contains the topological current. This is easy to see, as the topological current is the field strength associated with the potential, and so appears at two orders of $\theta$ above the potential. Explicitly, applying two supersymmetry transformations produces a momentum generator, and so

$$
\begin{aligned}
\left\{Q_{\alpha}, \tilde{S}_{\mu_{1}}^{\alpha}\right\} & =\partial_{\alpha}^{\beta} A_{\mu_{1} \cdots \mu_{d-2}}\left(\gamma^{\mu_{2}} \cdots \gamma^{\mu_{d-2}}\right)_{\alpha}^{\beta}+\cdots \\
& \supset \epsilon_{\mu_{1} \cdots \mu_{d}} \partial^{\mu_{2}} A^{\mu_{3} \cdots \mu_{d}}=J_{\mu_{1}}
\end{aligned}
$$

and thus the topological current appears in the Lorentz decomposition of $\left\{Q_{\alpha}, \tilde{S}_{\mu}^{\alpha}\right\}$. Since the $N=1$ supersymmetry transform of $\tilde{S}$ contains the topological current, then $\tilde{S}$ must not only be non-trivial but must be distinct from the original supercurrent [15]. Thus the charge associated with $\tilde{S}$ is a new conserved spinor charge which transforms into the 
topological charge under the original supersymmetry, and so this theory has an $N=2$ supersymmetry invariance in which the topological charge appears as a central charge. This completes the argument, and we have shown what we wished to show in this section.

We make four comments to conclude this section. First, there may be other non-trivial terms in the $N=1$ supersymmetry transform of $\tilde{S}$; this in fact generically occurs in even spacetime dimensions where one can have scalar and pseudoscalar central charges [10] [16]. In (2.4), we have explicitly identified only the terms where the topological charge appears. Second, note that we are not asserting that there is a canonical $N=2$ field content, but rather that with the $N=1$ field content, there is already a second supersymmetry (as happens in various of the specific models people have studied). Third, it is possible that our construction will produce a non-local $\tilde{S}_{\mu}^{\alpha}$ (stemming at heart from the possibility of a non-local solution to (A.2) (see the appendix)). This is not significant. The algebraic constraints on a theory are of course every bit as real whether they come from a conserved charge associated with a local or non-local conserved current. Furthermore, if a non-local solution does occur, this should merely reflect the absence of a sufficient number of auxiliary fields. And fourth and finally, notice that our index manipulations explicitly break down in $1+1$ dimensions. This makes sense. The potentials for topological currents in $1+1$ dimensions are scalars, and so there is no gauge transformation for such a potential, no way to require such a potential to transform into a divergenceless spinor current. Indeed, in $1+1$ dimensions, it is algebraically possible for $N=1$ supersymmetry to include central charges, so this case is expected to be different. In fact, explicit examples are constructed in [10], demonstrating that $1+1$ dimensional models need not have extended supersymmetry in the presence of topological charges and that the topological charges themselves need not appear as the central charges even in the $N=1$ supersymmetric case. Consequently, we find the distinction that our argument naturally draws between $1+1$ and higher dimensions especially compelling, as this reflects exactly the evidence accumulated from the studies of numerous specific models, as well as what is known from the general study of supersymmetry algebras.

\section{Bogomol'nyi Equations and Inequalities}

We have now established that (in $2+1$ or more spacetime dimensions) $N=$ 1 supersymmetric theories with a topological charge automatically have $N=2$ supersymmetry in which the topological charge appears as the central charge. What does this imply for the properties of topologically non-trivial field configurations? We will take up the cases of solitons and instantons in turn. 


\subsection{The Case of Solitons}

To begin our consideration of Bogomol'nyi relationships for solitons, we note that the result of the preceding section implies that supersymmetric theories with topological solitons necessarily exhibit Bogomol'nyi bounds and equations [10], as a consequence of the representation theory of extended superalgebras [17]. In such an extended superalgebra, the energy of a field configuration is bounded from below by the magnitude of its central charge [18], in this case the topological charge. This is the Bogomol'nyi bound. Furthermore, a field configuration which saturates such a bound is necessarily annihilated by a linear combination of supercharges. This algebraic condition serves the role of the Bogomol'nyi equation. Since supercharges can generically be represented by first order differential operators (first-order in spacetime, non-linear in field space), this means that field configurations that saturate a Bogomol'nyi bound in a supersymmetric theory satisfy a Bogomol'nyi equation.

Second, this in turn implies that any theory, supersymmetric or not, with topological solitons exhibits Bogomol'nyi relationships [7] [9]. We review the idea briefly here. Consider a theory with a topological charge $T_{0}$ and energy functional of the theory $E_{0}$. Now construct a supersymmetric extension of this theory. Note that any field configuration of the original theory is automatically a field configuration of the supersymmetric extension. Furthermore, since a topological charge is conserved without using the equations of motion, the extended theory has a topological charge $T_{s}$ that is identical to the topological charge $T_{0}$ of the original theory. Finally, it is generally possible to construct a supersymmetric extension such that the energy of any field configuration of the original theory is the same whether evaluated in the original theory (call this energy $E_{0}$ ) or its supersymmetric extension (call this energy $E_{s}$ ) [9]. (We will have more to say on this point shortly.)

Now consider a field configuration of the original theory, and evaluate its energy and topological charge in both the original and extended theories. We see immediately, then, that $T_{0}=T_{s}$ and $E_{0}=E_{s}$. But we know that $E_{s} \geq\left|T_{s}\right|$ for any field configuration of

1 Sometimes the simple application of the method described in Section 2 for obtaining the second supercurrent initially will yield a non-local equation here. This can in general be remedied by adding appropriate auxiliary fields. Such a use of auxiliary fields is already familiar from more conventional treatments of solitons even outside supersymmetric models, as exemplified by the gauge fields in the $2+1$ dimensional $\mathbf{C P}^{\mathbf{1}}$ model or abelian Chern-Simons model with vortices. 
the supersymmetric extension, due to the extended superalgebra that appears in a model. Thus for any field configuration of the original theory, we have the Bogomol'nyi bound

$$
E_{0} \geq\left|T_{0}\right|
$$

Furthermore, if a field configuration of the original theory satisfies $E_{0}=\left|T_{0}\right|$, then it also satisfies $E_{s}=\left|T_{s}\right|$ when viewed as a field configuration of the supersymmetric extension. Now any field configuration with $E_{s}=\left|T_{s}\right|$ satisfies a Bogomol'nyi equation in the supersymmetric theory. But for the field configuration in question, this equation involves only the fields of the original theory, and thus this equation is the Bogomol'nyi equation of the original theory, satisfied by any field configuration for which $E_{0}=\left|T_{0}\right|$. (Elimination of the extra superpartners or auxiliary fields introduces no additional derivatives.) Thus the requirement that a theory with a topological charge have a consistent supersymmetric extension implies that a theory with a topological charge - even a non-supersymmetric theory — necessarily exhibits Bogomol'nyi relationships.

It is worthwhile to make some comments regarding the construction of supersymmetric extensions for which $E_{0}=E_{s}$ for any field configuration of the original theory. One might be concerned that the supersymmetric extension of a theory would have new, additional interactions involving only the fields of the original theory, which would then invalidate this equality between the two energy functionals for the field configurations of interest. (Obviously interactions involving both original and extra fields pose no problem, as they vanish when the extra fields vanish.) As we discuss below, this is not a problem.

The reason this is not a problem is that we do not need to find some minimal supersymmetric extension; any supersymmetric extension, even one with a large number of additional fields and one that is non-renormalizable, suffices. Thus, it is only necessary that there be some supersymmetric extension with the desired equality between the two energy functionals.

For example, suppose the original theory has a Yukawa coupling. If we embed the scalar and the fermion in the same superfield, supersymmetry will require a quartic scalar self-interaction that is not present in the original theory. Instead, however, for our purposes, we choose a supersymmetric extension with two superfields, one of which contains the original scalar and the other of which contains the original fermion. This ensures that, for example, the quartic scalar interactions required by supersymmetry in this case (after elimination of auxiliary fields) always involve some of the additional fields 
not present in the original theory, and so these terms make no contribution to the energy when the field configuration is a field configuration of the original theory.

We can merge this approach with a technique introduced in [9] for constructing appropriate supersymmetric extensions of scalar potentials to produce a general procedure for obtaining a supersymmetric extension of the original Lagrangian with a suitable energy functional. The prescription below depends on certain dimension-independent features of supersymmetry, such as superfields, supercovariant derivatives, and auxiliary fields. It details a systematic and comprehensive (if sometimes cumbersome) procedure for obtaining an appropriate supersymmetric generalization that meets our requirements, although often one need not invoke this somewhat elaborate construction.

Suppose we have a some Lagrangian terms $\mathcal{L}_{\text {int }}$ which we wish to extend in a suitable supersymmetric fashion. (As our notation suggests, in a typical application these would be interaction terms, although there is nothing in principle to prevent one from applying this to any subset or even all of the terms in a given Lagrangian, and so this notation should not be taken too literally.) For each field included in these interactions, we associate a superfield. Then by inserting appropriate supercovariant derivatives if necessary (so as to project out the desired Lorentz components of the superfields), one can obtain a superfield for each original field in which the original field appears as the lowest (i.e., $\theta=0)$ component. We will call this superfield the associated superfield of a given field. For example, in $3+1$ dimensions, one could place a fermionic field in a scalar superfield $\Phi$; then $D_{\alpha} \Phi$ would be the associated superfield, since it has the fermion as its lowest component. Alternatively, one might choose as the associated superfield a fermionic superfield, a chiral superfield endowed with a spinor index, which already has the fermion as its lowest component.

Note that in this construction, one would generally view a complex field $S+i P$ in the original theory as two separate real fields. One then introduces two associated superfields in the extended theory, one for $S$ and one for $P$. Depending on the dimension, it may be necessary to take each of these associated superfields to be complex, so that a single complex field in the original theory is enlarged into two complex superfields in the extended theory.

Replacing each field in $\mathcal{L}_{\text {int }}$ by its associated superfield, one obtains a superfield expression $\mathcal{L}_{\text {int }}^{s}$ which has $\mathcal{L}_{\text {int }}$ as its lowest (i.e., $\theta=0$ ) term. We also introduce an additional superfield $\Xi$, along with a quadratic term for $\Xi$, and a term coupling $\Xi$ to 
$\sqrt{\mathcal{L}_{\text {int }}^{s}}$ in the superpotential.2 The idea is that $\Xi$ has some auxiliary field, call it $B$, so that in terms of components, the supersymmetric Lagrangian contains both a $B^{2}$ term and a $B \sqrt{\mathcal{L}_{\text {int }}}$ term. Eliminating the auxiliary field $B$, one reproduces $\mathcal{L}_{\text {int }}$ of the original theory. The other terms in the supersymmetric Lagrangian involve the other non-auxiliary components of $\Xi$ as well as other components of the associated superfields, and so vanish when we set the extra physical fields of the extended theory to zero. Thus when the additional fields vanish, the Lagrangian, and hence the energy functional, too, for the supersymmetric theory reduces to that of the original theory.

Obviously, to implement this, one needs to use superfields appropriate to the given dimension [19]. However, because we are not restricted to some particular supersymmetric extension, it is in fact very easy to formulate a supersymmetric extension with the desired properties in a dimension independent way.

To begin to understand the flexibility of such a construction, it is useful first to consider the familiar case of $3+1$ dimensions. Here, one can use in our construction $\int d^{4} \theta \Xi^{*} \Xi$ as the quadratic term, where $\Xi$ is a chiral superfield which has its auxiliary field at $\mathcal{O}\left(\theta^{2}\right)$, and $\int d^{2} \theta \sqrt{\mathcal{L}_{\text {int }}^{s}} \Xi+$ h.c. as the superpotential term. This formulation is not unique, however. We can introduce a potential superfield $\Psi$, for example, with the identification $\Xi=\bar{D}^{2} \Psi$. The auxiliary field which appeared at order $\theta^{2}$ in $\Xi$ appears at order $\theta^{4}$ in $\Psi$. The quadratic term is of the form $\int d^{4} \theta D^{2} \bar{\Psi} \bar{D}^{2} \Psi$, while the superpotential term is $\int d^{4} \theta \Psi \sqrt{\mathcal{L}_{\text {int }}^{\text {s }}}+$ h.c. Notice that the field $\Psi$ is not subject to any restrictions (and does not correspond to an irreducible representation of the supersymmetry algebra).

We now generalize this second formulation to arbitrary dimension. Suppose we are considering a theory in $d$ spacetime dimensions, in which the superspace has a spinor coordinate $\theta^{\alpha}$ with $2 s$ components. If we consider a scalar superfield $\Xi$ in this superspace, its highest component is a scalar field $B$, which will be the auxiliary field we will use. The necessary quadratic term is $\int d^{2 s} \theta D^{s} \Xi D^{s} \Xi$, while the superpotential term that generates the interaction is $\int d^{2 s} \theta \sqrt{\mathcal{L}_{\text {int }}^{s}} \Xi$. This generates the necessary Lagrangian terms. Additional terms are also generated, but these all vanish when the other fields in $\Xi$ (all of which appear dynamically in these superspace action terms) are set to zero. 3 In this way,

2 The square root poses no problems; a Taylor series in Grassman quantities terminates. If $\mathcal{L}_{\text {int }}^{s}$ has no terms devoid of fermions, one can simply use $1+\mathcal{L}_{\text {int }}^{s}$ instead. Since our work is entirely classical, renormalizability is of no concern to us.

3 Of course, the superfield written down here need not correspond to an irreducible supersymmetry representation. This is not a problem for our construction, although if one wishes, one can project out the irreducible representation that includes the auxiliary field $B$. 
then, we see that it is always possible to construct a supersymmetric generalization of a given field theory, with the property that when the extra physical fields added vanish, the energy functional of the extended theory reduces to that of the original theory.

In practice, one need not necessarily invoke this construction, even in cases in which the minimal supersymmetric extension might seem to impose additional and unacceptable relations among the component fields. For example, consider a non-abelian gauge theory with a scalar field, say in the adjoint representation, as occurs in monopole theories. Coupling a scalar superfield to a vector superfield in a minimal way does produce additional scalar potential terms (the so-called $D$-terms), but it turns out that these automatically vanish when one sets the extra component fields to zero [9]. Thus, the reader should remember that on the one hand, we have obtained a general construction that demonstrates that is is always possible to define a suitable supersymmetric extension, but that on the other hand, many times a minimal supersymmetric extension will be satisfactory for our purposes.

\subsection{The Case of Instantons}

We now move to a consideration of instantons, demonstrating that if topological solitons generically exhibit Bogomol'nyi relationships, then instantons, too, generically exhibit action bounds, as well as "self-duality" equations when these action bounds are saturated.

Consider a $d$-dimensional theory with topological instantons. This means that the Euclidean theory, with action $S_{d}$, has field configurations with non-zero instanton number $I_{d}$. The instanton number reflects the topological classification of the finite action field configurations over $d$-dimensional Euclidean space. Field configurations of different instanton number are in different topological sectors.

Suppose we enhance this $d$-dimensional theory by adding a time coordinate and extra fields as necessary (e.g., timelike components for fundamental vector fields) to make a Lorentz invariant $d+1$-dimensional Minkowskian theory 4 This step is essential to our argument. Consequently, if the original action has dimension-specific expression, such as a term that can only be written with an epsilon tensor, our argument will not apply. However, it is only the appearance of such an expression in the action that poses a problem;

4 We also adjust an overall sign, so the Euclidean action is positive semi-definite, while the corresponding terms appear with the opposite sign in the Minkowski action. 
an epsilon tensor in the definition of the topological index, for example, poses no obstacle at all.

Now obviously, the instanton number becomes a conserved topological charge of the enhanced theory. The topological charge arises from the topological classification of finite energy field configurations over the $d$ dimensional Euclidean space within the $d+1$ dimensional Minkowski spacetime. Thus the topological classification of field configurations in the Euclidean theory becomes a topological classification in the $d+1$ dimensional theory, in which the added field components play no role. (Only the fields present in the original theory appear in the definition of the topological charge.) Since this classification of field configurations is topological, under time evolution, the topological classification of the field configuration in space cannot change, independent of the equations of motion, and so the topological classification that led to an instanton number in $d$ dimensions leads to a topological conserved charge in $d+1$ dimensions. As we have argued above, then, this $d+1$ dimensional theory necessarily exhibits a Bogomol'nyi bound.

Furthermore, in the dimensional enhancement, we need introduce no interactions different from the ones in the original theory, although those interactions may involve larger fields (e.g., four-component vs. two-component spinors). Consequently, when these additional field components are set to zero, the enhanced theory is of the same form as the original theory, except that all the fields have a time dependence as well. In the case that these fields are static, then, the energy $E_{d+1}$ of the enhanced theory and the Euclidean action of the original theory $S_{d}$ are identical.

Now consider an instanton configuration of the original theory. Clearly, we can also interpret it as a field configuration of the enhanced theory in which the additional fields all vanish and in which the non-zero fields are static. For such a field configuration, the topological charge $T_{d+1}$ in the enhanced theory is identically the instanton number $I_{d}$ in the original theory. Such a field configuration interpreted in the $d+1$ dimensional theory has non-zero topological charge, and as such, cannot violate the Bogomol'nyi bound of that theory. Hence, $E_{d+1} \geq\left|T_{d+1}\right|$. But, since for these field configurations, $E_{d+1}=S_{d}$ and $T_{d+1}=I_{d}$, it follows that

$$
S_{d} \geq\left|I_{d}\right|
$$

If the instanton saturates the bound (3.2), then not only is $S_{d}=\left|I_{d}\right|$, but, interpreted in the enhanced theory $E_{d+1}=\left|T_{d+1}\right|$. For field configurations for which $E_{d+1}=\left|T_{d+1}\right|$, we know that our previous analysis produces a Bogomol'nyi equation that this configuration 
must satsify. Since this configuration is necessarily static, this Bogomol'nyi equation which is satisfied in $d+1$ spacetime dimensions is actually an equation in $d$ dimensional space. This equation is an equation in $d$ dimensional Euclidean space satisfied by any instanton configuration that saturates the action bound (3.2). In other words, it is the "self-duality" equation for saturating instantons.

Thus, whenver a theory with instantons can be enlarged to Minkowski theory in one higher dimensions - which is the typical case - those instantons will exhibit Bogomol'nyi relationships. In fact, even in a theory with a dimension-dependent term, one might still be able to employ our results. Consider a Lagrangian which contains a set of terms that can be dimensionally enhanced, plus a single term which cannot be. One first obtains the Bogomol'nyi relationships for the theory without this additional problematic term using our general methods. Then one can focus specifically on the consequences of the additional term to see how it modifies the Bogomol'nyi relationships already obtained.

Note by the way that we have not claimed that any soliton which solves the equations of motion in $d+1$ dimensions becomes, upon dimensional reduction, an instanton which solves $d$ dimensional equations of motion. An obvious counterexample is provided by a time-dependent soliton of the $d+1$ dimensional theory. Importantly, here we are working in the other direction, and an instanton of the $d$ dimensional theory is always a field configuration of the enhanced $d+1$ dimensional theory that we have defined.

\section{Closing Comments}

In this paper, we have significantly improved on our earlier analysis of topological solitons. We have extended our model-independent approach to include instantons as well as solitons, and to include solitons and instantons in $2+1$ or more and two or more dimensions, respectively. Most instructively, our analysis of the appearance of extended supersymmetry in models with solitons clearly demonstrated the difference between topological conservation laws in $1+1$ and higher dimensional supersymmetric theories. The very basic and essential way that our analysis breaks down below $2+1$ dimensions - just as the theoretical evidence requires - we find as telling evidence that our explanation for the appearance of extended superalgebras in theories with solitons is based on the truly fundamental aspects of the problem.

In closing, we remark that we have used supersymmetry in quite a powerful way to understand non-supersymmetric field theories. Moreover, our methods are constructive, 
not just formal, and give, to our knowledge, the only model-independent approach to the Bogomol'nyi -type properties of solitons and instantons. We note that this use of supersymmetry gives a systematic approach to index theorems in instanton and soliton backgrounds, and to demonstrating equivalences between the bosonic and fermionic excitation spectra in such backgrounds. We are currently investigating these matters.

One refinement of our results would be the identification of a way to use supersymmetry directly to obtain results about instantons, rather than by going through the process of dimensional enhancement. We imagine that this is possible, but so far have been unsuccessful in our attempts to do so.

\section{Acknowledgments}

D.S. acknowledges the support of a Hobart and William Smith Faculty Research Grant and of NSF Grant No. PHY-9207859. D.S. also thanks the LNS Theory Group at Cornell University, where much of this research was carried out, for its hospitality.

\section{Appendix A.}

Here we show that, by means of a gauge transformation, it is always possible to choose the potential $A$ of (2.1) to be divergenceless In the case of $2+1$ dimensions, this gauge is simply Lorentz gauge. In the case of $3+1$ dimensions, one can argue this by formal equivalence to an electrodynamics problem. Let $A^{\mu \nu}$ be a rank-2 antisymmetric tensor. Now interpret $j^{\nu}=-\partial_{\mu} A^{\mu \nu}$ as an electromagnetic current, and let $F^{\mu \nu}$ be the electromagnetic field generated by this current. This field strength can, of course, be written as the "curl" of some vector potential. Thus $A_{\mu \nu}^{\prime}=A^{\mu \nu}+F^{\mu \nu}$ is divergenceless, antisymmetric, and gauge equivalent to $A^{\mu \nu}$. In higher dimensions, one can make similar physical arguments showing the existence of such a gauge.

Alternatively, one can use an $i \epsilon$ prescription. Let $A^{\prime}=A+\partial \Omega$. (We suppress indices for simplicity where possible.) We now show that we can choose $\Omega$ such that $\partial \cdot A^{\prime}=0$. Note first that, without loss of generality, we may take $\Omega$ to be divergenceless. One can argue this by induction. We know that a vector potential can be gauged to be divergenceless. Now consider the gauge transformations of an antisymmetric 2-tensor. Such transformations are labeled by gauge equivalence classes of vectors, and so without loss of generality we can consider only the gauge transformations given by divergenceless vector potentials. One uses 
this as in the argument below to show that one can always gauge an antisymmetric 2-tensor to be divergenceless. And now one uses this result in considering the gauge transformations of antisymmetric 3 -tensors, which are labeled by gauge equivalence classes of antisymmetric 2-tensors, etc.

Considering only divergenceless $\Omega$, we see that for $A^{\prime}$ to be divergenceless, we must have

$$
\partial^{2} \Omega^{\mu_{2} \cdots \mu_{d-2}}=\partial_{\mu_{1}} A^{\mu_{1} \cdots \mu_{d-2}} .
$$

Making an $i \epsilon$ prescription, we can analytically continue (A.1) to

$$
\left(\partial^{2}+i \epsilon\right) \Omega^{\mu_{2} \cdots \mu_{d-2}}=\partial_{\mu_{1}} A^{\mu_{1} \cdots \mu_{d-2}} .
$$

For $\epsilon \neq 0, \partial^{2}+i \epsilon$ is invertible, so $\Omega=\lim _{\epsilon \rightarrow 0}\left(\partial^{2}+i \epsilon\right)^{-1} \partial A$. With this choice of $\Omega, A^{\prime}$ is divergenceless. 


\section{References}

[1] R. Rajaraman, Solitons and Instantons, Amsterdam, North-Holland, 1987.

[2] E.B. Bogomol'nyi, Sov. J. Nucl. Phys. 24 (1976) 449.

[3] G. 't Hooft, Phys. Rev. D14 (1976) 3432;

A.M. Polyakov, JETP Lett. 20 (1974) 194.

[4] M.K. Prasad and C.H. Sommerfield, Phys. Rev. Lett. 35 (1975) 760.

[5] A.A. Belavin, A.M. Polyakov, A.S. Schwartz, and Yu.S. Tyupkin, Phys. Lett. 59B (1975) 85.

[6] G. 't Hooft, Phys. Rev. Lett. 37 (1976) 8;

G. 't Hooft, Phys. Rev. D14 (1976) 3432;

A.M. Polyakov, Nucl. Phys. B121 (1977) 429.

[7] Z. Hlousek and D. Spector, Mod. Phys. Lett. A7 (1992) 3403;

D. Spector, Bogomol'nyi Explained: An Application of $N=0$ Supersymmetry, in Particle Physics from Underground to Heaven: the Proceedings of the 15th Johns Hopkins Workshop on Current Problems in Particle Theory, World Scientific, 1992).

[8] Z. Hlousek and D. Spector, Nucl. Phys. B370 (1992) 143.

[9] Z. Hlousek and D. Spector, Bogomol'nyi Explained, Nucl. Phys. B397 (1993) 173.

[10] D. Olive and E. Witten, Phys. Lett. 78B (1978) 97.

[11] C. Lee, K. Lee, and E. J. Weinberg, Phys. Lett. 243B (1990) 105.

[12] Z. Hlousek and D. Spector, Nucl. Phys. B344 (1990) 763;

see also E. Witten, Phys.Rev. D10 (1977) 2991, and the first item in [7].

[13] J.A. de Azcarraga, J.P. Gauntlett, J.M. Izquierdo, and P.K. Townsend, Phys. Rev. Lett. 63 (1989) 2443;

M. Cvetic, F. Quevedo, and S.-J. Rey, Phys. Rev. Lett. 67 (1991) 1836.

[14] C. Cronström and J. Mickelsson, J. Math. Phys. 24 (1983) 2528;

R. Jackiw in S. Treiman, R. Jackiw, B. Zumino, and E. Witten Current Algebra and Anomalies (World Scientific, Singapore, 1985).

[15] R. Haag, J. Łopuszański, and M. Sohnius, Nucl. Phys. B288 (1975) 257.

[16] Z. Hlousek and D. Spector, Phys. Lett. 283B (1992) 75.

[17] A. Salam and J. Strathdee, Nucl. Phys. B80 (1974) 499.

[18] S. J. Gates, M.T. Grisaru, M. Roček, and W. Siegel, Superspace, or One Thousand and One Lessons in Supersymmetry (Benjamin/Cummings, Reading MA, 1983);

J. Wess and J. Bagger, Supersymmetry and Supergravity (Princeton University Press, Princeton, NJ, 1983).

[19] W. Siegel, Phys. Lett. 80B (1979) 220 ;

B.E.W. Nilsson, Nucl. Phys. B174 (1980) 335;

L.B. Litov, Preprint JINR-E2-83-467-mc, July 1983, submitted to Bulg. Phys. J.; X.A. Zhou, Can. J. Phys. 66 (1988) 757. 